\title{
Terahertz Emission and Absorption Characteristics of Silicon Containing Boron and Phosphorous Impurity Dopants and the Effect of Temperature
}

\author{
Guy Matmon*, Stephen A. Lynch*, Paul Townsend*, Douglas J. Paul*, Mike Bain ${ }^{\dagger}$, Harry S. Gamble ${ }^{\dagger}$, \\ Jing Zhang ${ }^{\ddagger}$, Zoran Ikonic ${ }^{\S}$, Robert W. Kelsall ${ }^{\S}$, and Paul Harrison ${ }^{\S}$ \\ *University of Cambridge, Cavendish Laboratory, Madingley Road, Cambridge CB3 0HE, UK. \\ Telephone: 00441223 764166, Email: gm331@cam.ac.uk \\ ${ }^{\dagger}$ Queens University, School of Electrical and Electronic Engineering, Ashby Building, Stranmills Road, Belfast BT9 5AH, UK. \\ ${ }_{\ddagger}^{\ddagger}$ Imperial College, EXSS, Blackett Laboratory, Imperial College London, Prince Consort Road, London SW7 2BW, UK. \\ ${ }^{\S}$ University of Leeds, Institute for Microwaves and Photonics, School of Electronic and Electrical Engineering, UK.
}

\begin{abstract}
The emission and absorption characteristics of boron-doped and phosphorous-doped silicon at terahertz frequencies are investigated. Different doping concentrations are considered and individual terahertz optical transitions are identified. The effect of temperature is considered.

\section{INTRODUCTION}

Semiconductor lasers are gaining ever increasing importance in industrial applications and there have been sustained efforts over the past few years to develop a silicon based laser source. Such a device would represent a considerable advancement in optoelectronic technology since it would open the way for inexpensive monolithic integrated optical and electronic components. In the last two years there has been considerable interest in impurity dopants such as boron and phosphorous since it was demonstrated that such materials can show emission at $\mathrm{THz}$ frequencies [1]-[5]. In this paper we investigate the temperature dependence of these impurity transitions in order to estimate the most optimistic operating range of an impurity based silicon laser. These results should also be taken into account when designing potential $\mathrm{THz}$ silicon waveguides since both boron and phosphorous are commonly used to fabricate $\mathrm{n}$ - and $\mathrm{p}$-type Ohmic contacts and could contribute to potential optical losses.
\end{abstract}

\section{EXPERIMENTAL}

Figure 1 shows two $\mathrm{THz}$ electroluminescence spectra for (a) a sample containing boron and (b) a sample containing phosphorous. Figure 1 shows several strong sharp spectral features in the electroluminescence spectrum which can be identified by comparison with absorption spectra in [7]. In the boron doped sample impurity based electroluminescence was extinguished at temperatures above $4.2 \mathrm{~K}$, while in the case of the phosphorous doped sample, impurity based electroluminescence was observed up to about $40 \mathrm{~K}$.

In order to further assess the effect of temperature and dopant concentration on these transitions, we performed a series of absorption measurements on bulk doped silicon

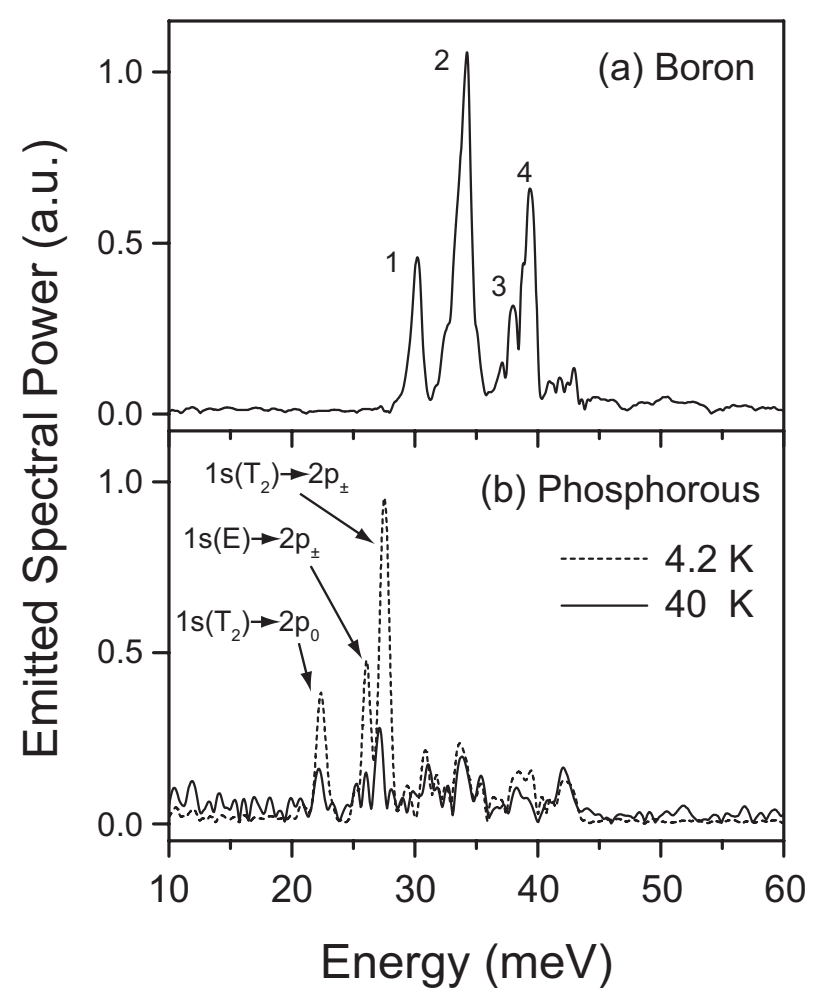

Fig. 1. THz electro-luminescence spectra for (a) a sample containing boron and (b) a sample containing phosphorous.

wafers. The details of each wafer are summarized in table I. Phosphorous doped wafers are labeled P1-P4 and boron doped wafers are labeled B1-B3. Samples were attached to the cold finger of a continuous flow cryostat allowing temperature tuning in the range $4.2 \mathrm{~K} \leq \mathrm{T} \leq 300 \mathrm{~K}$. Measurements were made using a Bruker 66VS spectrometer and a QMC helium cooled composite Si-bolometer detector.

The total absorption of each wafer was first measured as a 
TABLE I

WAFER LABELS AND CORRESPONDING RESISTIVITY AND DOPING CONCENTRATIONS (P-PHOSPHOROUS AND B-BORON).

\begin{tabular}{|c|c|c|}
\hline Wafer label & $\begin{array}{c}\text { Resistivity } \\
(\Omega \mathrm{cm})\end{array}$ & $\begin{array}{c}\text { Doping Conc. } \\
\left(\mathrm{cm}^{-3}\right)\end{array}$ \\
\hline P1 & $0.005-0.018$ & $1 \times 10^{19}$ \\
P2 & $0.04-0.14$ & $2 \times 10^{17}$ \\
P3 & 0.3 & $1.5 \times 10^{16}$ \\
P4 & $2-9$ & $5 \times 10^{15}$ \\
B1 & 0.02 & $5 \times 10^{18}$ \\
B2 & 0.05 & $1 \times 10^{18}$ \\
B3 & $10-20$ & $1 \times 10^{15}$ \\
\hline
\end{tabular}

function of temperature. No transmitted signal was recorded through samples with dopant concentrations greater than $10^{18}$ $\mathrm{cm}^{-3}$, and very little signal was recorded through the next highest dopant concentration. The behavior of the samples with dopant concentrations in the range $10^{15}-10^{16} \mathrm{~cm}^{-3}$ is markedly different. Figure 2 shows the total absorption as a function of temperature of sample $\mathrm{P} 3$ (phosphorous) and sample B3 (boron), in the temperature range $4.2 \mathrm{~K} \leq \mathrm{T} \leq 200 \mathrm{~K}$. The total absorption for boron does not show a large change

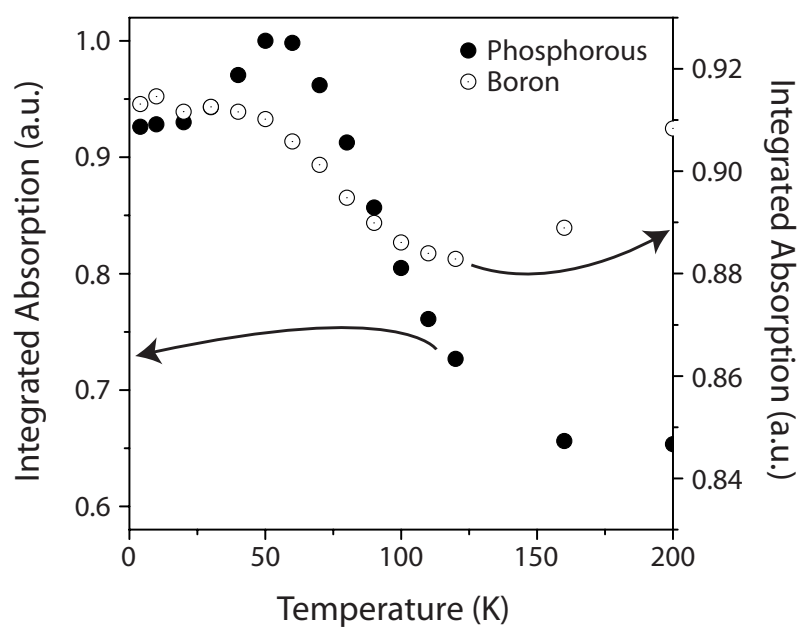

Fig. 2. Integrated absorption for samples P3 (phosphorous at $1.5 \times 10^{16}$ $\mathrm{cm}^{-3}$, and $\mathrm{B} 3$ (boron at $1 \times 10^{15} \mathrm{~cm}^{-3}$ ), in the spectral range $100-500 \mathrm{~cm}^{-1}$.

over the temperature range in question. The maximum absorption is at $4.2 \mathrm{~K}$ and the absorption decreases with increasing temperature up to about $100 \mathrm{~K}$. This behavior can be easily explained with reference to figure 3 . At very low heat sink temperatures three very sharp absorption features can be observed between 30-40 meV. These correspond lines 1, 2, and 4 of the well-known $p_{3 / 2}$ series of $\operatorname{Si}(\mathrm{B})$ [7], [8]. As the heatsink temperature is raised between $4.2 \mathrm{~K}$ and $90 \mathrm{~K}$, the transitions become progressively weaker, until they are finally extinguished around $100 \mathrm{~K}$. This corresponds closely with a reduction in the total absorption up to $120 \mathrm{~K}$ as shown in figure 2 . In contrast, the behavior of the phosphorous doped sample in figure 2 is quite different. Unlike the boron doped sample,

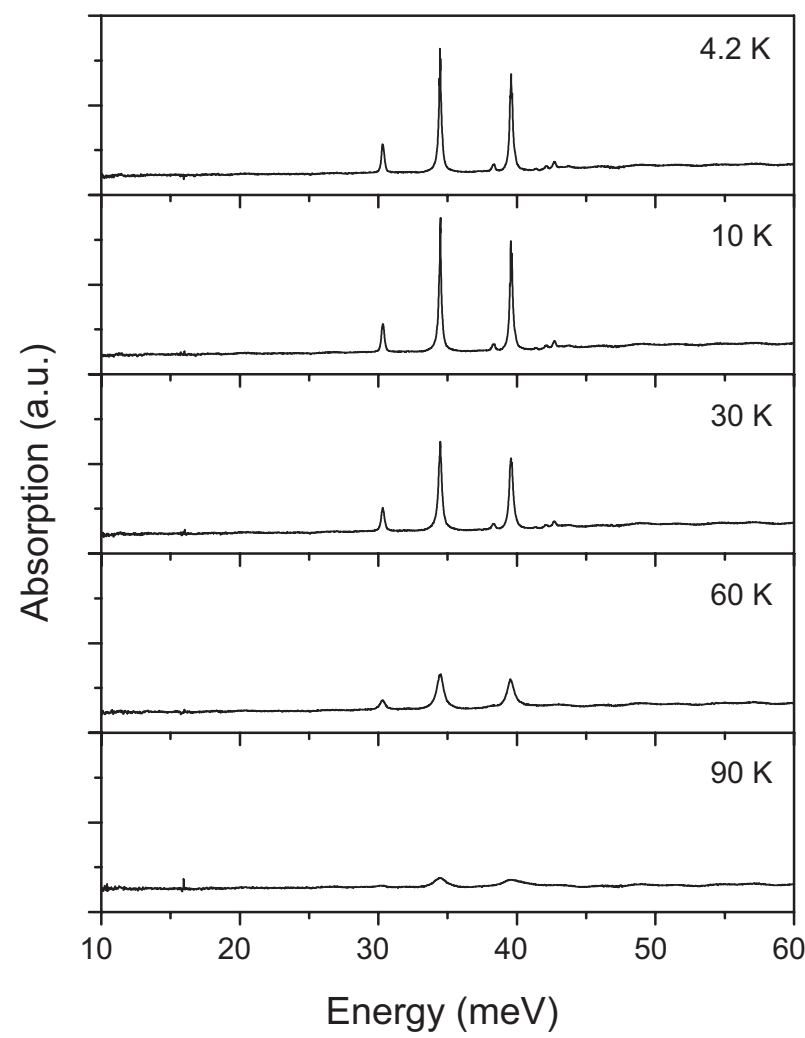

Fig. 3. THz absorption from boron doped silicon wafer B3 with temperature. The wafer has a resistivity of $10-20 \Omega \mathrm{cm}$, giving a boron concentration of $1 \times 10^{15} \mathrm{~cm}^{-3}$.

the maximum absorption of the phosphorous doped sample is not at $4.2 \mathrm{~K}$, and there is a local maximum in the absorption with temperature curve at $50 \mathrm{~K}$. Close examination of figure 4 reveals that the absorption spectrum is dominated by three features in the range $30-45 \mathrm{meV}$ at $4.2 \mathrm{~K}$. These correspond to the $2 p_{0}, 2 p_{ \pm}$, and $3 p_{ \pm}$Lyman series transitions to the ground state $1 s\left(A_{1}\right)$ [7]. As the heatsink temperature is increased, a further set of thermally activated absorption lines appear between 20-30 meV. By considering the binding energies in [7], it is possible to show that these transitions are between the $2 p_{0}, 2 p_{ \pm}$energy levels and the excited ground state $1 s\left(T_{1}\right)$. These features reach maximum transition strength at $50 \mathrm{~K}$, the local maximum of the relevant absorption with temperature curve in figure 2 . It is from these additional thermally activated transitions that emission was observed in figure 1 . Above $50 \mathrm{~K}$, the strength of all the transitions begins to weaken until they are extinguished at around $100 \mathrm{~K}$.

\section{CONCLUSION}

The temperature dependence of $\mathrm{THz}$ optical transitions from common dopants boron and phosphorous has been investigated. Sharp spectral features at $\mathrm{THz}$ frequencies resulting from these elements in single crystal silicon were best observed in samples with bulk dopant concentrations in the range $10^{15}-10^{16} \mathrm{~cm}^{-3}$. Above these dopant concentrations 


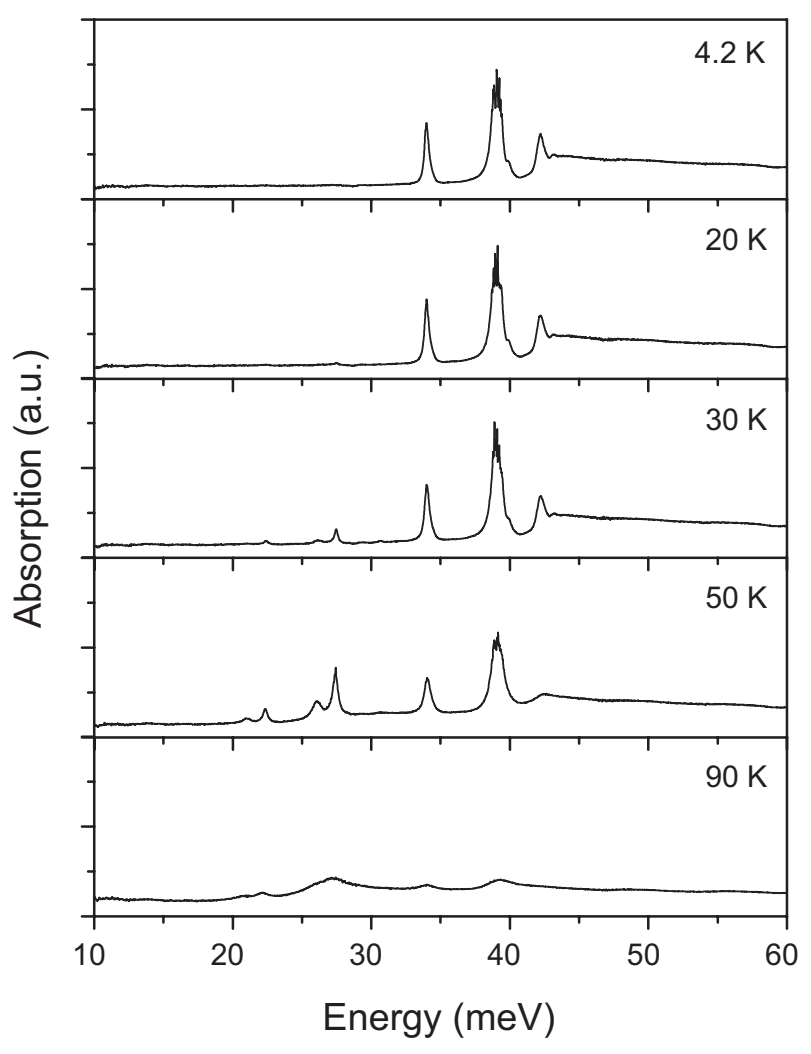

Fig. 4. THz absorption from phosphorous doped silicon wafer P3 with temperature. The wafer has a resistivity of $0.3 \Omega \mathrm{cm}$, giving a phosphorous concentration of $1.5 \times 10^{16} \mathrm{~cm}^{-3}$.

the spectral features become very much broadened as the wavefunctions become delocalized. Below $10^{15} \mathrm{~cm}^{-3}$ the absorption becomes rapidly weaker and the spectral features become difficult to measure. The overall absorption due to boron in silicon decreases monotonically with increasing temperature in the temperature range $4.2 \mathrm{~K} \leq \mathrm{T} \leq 100 \mathrm{~K}$, as the transition strength of the boron lines becomes weaker and the transitions are eventually extinguished. The overall absorption due to phosphorous in silicon exhibits a local maximum at $50 \mathrm{~K}$ and then decreases with increasing temperature up to $100 \mathrm{~K}$. This local maximum corresponds with the observation of thermally activated transition lines which are not observed at lower temperatures. In each case the exact transitions have been identified.
These results are important and need to be taken into account when designing potential $\mathrm{THz}$ silicon waveguides since both boron and phosphorous are commonly used to realize $\mathrm{n}$ - and $\mathrm{p}$-type Ohmic contacts and will contribute to potential losses. The measured temperature dependence of these transitions also shows that the most optimistic operating range of an impurity based laser is $4.2 \mathrm{~K} \leq \mathrm{T} \leq 90 \mathrm{~K}$. This represents an ideal passive device with no current flowing. In a real device with current flowing and associated Joule heating the actual operating range is likely to be much narrower and it is unlikely such a device would have a maximum operating temperature above $40 \mathrm{~K}$ as in figure 1 . This is quite consistent with the value quoted in [9].

\section{ACKNOWLEDGMENTS}

This research was supported by U.S. DARPA under Air Force contract F-19628-99-C-0074 and EU research grant SHINE IST-2001-38035.

\section{REFERENCES}

[1] S.A. Lynch, S.S. Dhillon, R. Bates, D.J. Paul, D.D. Arnone, D.J. Robbins, Z. Ikonic, R.W. Kelsall, P. Harrison, D.J. Norris, A.G. Cullis, C.R. Pidgeon, P. Murzyn, and A. Loudon. Si-based electroluminescence at THz frequencies. J. Mat. Sci. B, 89:12, 2002.

[2] R. Bates, S.A. Lynch, D.J. Paul, Z. Ikonic, R.W. Kelsall, P. Harrison, S.L. Liew, D.J. Norris, A.G. Cullis, W.R. Tribe, and D.D. Arnone. Interwell intersubband electroluminescence from si/sige quantum cascade emitters. Appl. Phys. Lett., 83(20):4092-4094, November 2003.

[3] T.N. Adam, R.T. Troeger, S.K. Ray, P.-C. Lv, and J. Kolodzey. Terahertz electroluminescence from boron-doped silicon devices. Appl. Phys. Lett., 83(9):1713-1715, September 2003.

[4] P.-C. Lv, R.T. Troeger, T.N. Adam, S. Kim, J. Kolodzey, I. N. Yassievich, M. A. Odnoblyudov, and M. S. Kagan. Electroluminescence at 7 terahertz from phosphorus donors in silicon. Appl. Phys. Lett., 85(1):22, July 2004

[5] P.-C. Lv, R.T. Troeger, S. Kim, S.K. Ray, K.W. Goossen, J. Kolodzey, I.N. Yassievich, M.A. Odnoblyudov, and M.S. Kagan. Terahertz emission from electrically pumped gallium doped silicon devices. Appl. Phys. Lett., 85(17):3660-3662, October 2004.

[6] M. Bain, H.A.W. El Mubarek, J.M. Bonar, Y. Yang, O. Buiu, H. Gamble, B.M. Armstrong, P.L.F. Hemment, S. Hall, and P. Ashburn. SiGe HBTs on bonded SOI incorporating buried silicide layers. IEEE Tran. Electron. Dev. 52(3):317-324, March 2005.

[7] C. Jagannath, Z.W. Grabowski, and A.K. Ramdas. Linewidths of the electronic spectra of donors in silicon. Phys. Rev. B, 23(5):2082-2098, March 1981.

[8] A.K. Ramdas and S. Rodriguez. Spectroscopy of the solid-state analogues of the hydrogen atom: donors and acceptors in semiconductors. Rep. Prog. Phys., 44:1297-1387, 1981.

[9] S.G. Pavlov, H.W. Hubers, M.H. Rummeli, R.Kh. Zhukavin, E.E. Orlova, V.N. Shastin, and H. Riemann. Far-infrared stimulated emission from optically excited bismuth donors in silicon. Appl. Phys. Lett., 80(25):47174719, June 2002. 